\title{
Biogenic synthesis of magnesium oxide nanoparticles using Manihot esculenta (Crantz) leaf extract
}

\author{
Enobong R. Essien ${ }^{1}$ (D) $\cdot$ Violette N. Atasie $^{1} \cdot$ Anastecia O. Okeafor $^{1} \cdot$ Davies O. Nwude $^{1}$
}

Received: 7 June 2019 / Accepted: 13 November 2019 / Published online: 23 November 2019

(c) The Author(s) 2019

\begin{abstract}
Recently, plant-mediated route or green approach for preparing metal and metal oxide nanoparticles has received enormous attention due to the ease of preparation and environmental friendliness when compared to physical and chemical methods. Plants contain phytochemicals which have been proposed as bio-reductants and capping agents for forming metal nanoparticles. Therefore, this study was aimed to prepare magnesium oxide nanoparticles (MgONPs) using aqueous extract of Manihot esculenta leaf. The leaf extract was first analyzed in a gas chromatograph-mass spectrometer (GC-MS) to examine the phytochemicals present. Then, the MgONPs formed were evaluated using UV-Visible (UV-Vis) spectrophotometry, scanning electron microscopy (SEM), energy-dispersive X-ray analysis (EDX), transmission electron microscopy (TEM), X-ray diffractometry (XRD) and Fourier transform infrared (FTIR) spectroscopy, to confirm the formation of MgONPs and to determine the morphology, elemental composition, shape and size, phase composition and nature of bonds present in the sample. Results revealed the formation of monodisperse, hexagonal shaped MgONPs of average size $36.7 \mathrm{~nm}$ having potentials for application in catalysis and as antimicrobial agent. Hence the process reported herein could be optimized for large-scale preparation of MgONPs.
\end{abstract}

Keywords Monodisperse $\cdot$ Manihot esculenta $\cdot$ Green synthesis $\cdot$ Magnesium oxide nanoparticles $\cdot$ Capping agent

\section{Introduction}

Nanotechnology is a promising field of Materials Science that is capable of producing materials of various types at the nanoscale level. Nanoparticles are a wide class of materials that include particulate substances having dimension 1-100 nm [1]. In recent times, metal nanoparticles research has attracted immense attention due to their unusual properties compared to bulk metals [2], because at the nanoscale, various physiochemical properties can be severely influenced. Metal nanoparticles have wide biomedical and pharmaceutical applications as alternative antimicrobial agents due to the reemergence of infectious diseases and the appearance of antibiotic-resistant strains. Magnesium nanoparticles (MgONPs), in particular, have been applied in catalysis, toxic waste remediation, paints, superconducting

Enobong R. Essien

reggiessien@gmail.com

1 Department of Chemical and Food Sciences, Bells University of Technology, PMB 1015, Ota, Ogun State, Nigeria products, optical, electrical, electronics, antiseptics, antibacterial activities, semiconductors and catalytic devices [3].

Generally, conventional chemical synthesis methods of nanoparticles, besides being expensive, pose various health and environmental risks [4] due to the toxic nature of some of the starting reagents and by-products associated with these methods.

Synthesis of metal nanoparticles using plant extracts is attracting increasing interest as an alternative approach. Biologically synthesized metal nanoparticles via plant-mediated pathway have the benefits of being facile, nontoxic and ecofriendly. Biomolecules present in plant extracts act as reducing and capping agents, forming stable nanoparticles. Thus, the properties of the obtained metal nanoparticles, such as biocompatibility and antimicrobial activity, also depend on the properties of the various phytochemicals present in the plant extracts from which they were synthesized.

There are a number of reports on the preparation of MgONPs via plant-mediated route. Jhansi et al. [5] prepared MgONPs in various nanometer sizes using white button mushroom aqueous extract and studied their effects on peanut (Arachis hypogaea $\mathrm{L}$.) seed germination. Of the different 
three sample sizes studied, the one with the least particle size gave the greatest enhancement in seed germination. This, they suggested was due the more efficient penetration of the MgONPs into the seed coat to promote $\mathrm{Mg}$ content and water uptake needed for germination. Abdallah et al. [6] synthesized $\mathrm{MgO}$ nano-flowers using aqueous extracts from Rosmarinus officinalis L. (Rosemary) flowers as the reducing agent. The obtained $\mathrm{MgO}$ nano-flowers showed significant antimicrobial effect against Xanthomonas oryzae pv. oryzae, Furthermore, in the report of Vergheese and Vishal [7], MgONPs were synthesized from aqueous leaf extract of Trigonella foenum-graceum and tested for its antimicrobial activity against the Gram-positive $E$. Coli, Gram-negative $S$. aereus and Bacillus bacteria. The MgONPs showed potency against E. Coli and S. aereus at a similar concentration, but in the case of Bacillus double dosage was required to achieve a similar effect.

Manihot esculenta is extensively cultivated as an annual crop in the tropical and subtropical regions of the world for its edible starchy tuberous root, a major source of food carbohydrates in the tropics. Cassava, the tubers from the plant, is a major staple food in the developing world, providing a basic diet for over half a billion people [8]. The leaf contains vitamins A, B1, calcium, calories, phosphorus, protein, fat, carbohydrate and iron. It can be used to treat measles, small pox, chicken pox, skin rashes, fevers and headaches, colds, constipation, ringworm, tumor, conjunctivitis, sores and abscesses [9]. Furthermore, chlorophyll in the leaves serves as antioxidant and anticancer agent. These health attributes of Manihot esculenta leaf may be attributed to the phytochemicals present.

Even though the tubers of Manihot esculenta serve as staple food for the teaming population in some developing countries where the crop is cultivated on a large scale, the leaf is not well known to serve as a major source of vegetable. Thus, it is often discarded as waste after harvesting the tubers. It is against this backdrop, therefore, and given the large-scale cultivation of this crop in many parts of the world, that the present work is designed to prepare MgONPs using Manihot esculenta leaf extract as the reducing agent towards a potential commercial scale, ecofriendly synthesis of MgONPs. To best of our knowledge there is no current report on the synthesis of MgONPs from Manihot esculenta leaf.

\section{Materials and methods}

\section{Materials}

The Manihot esculenta leaves used for this study were collected from the premises of Bells University of Technology,
Ota, Ogun State, South-west, Nigeria. $\mathrm{Mg}\left(\mathrm{NO}_{3}\right)_{2} \cdot 6 \mathrm{H}_{2} \mathrm{O}$ and absolute ethanol were purchased from Sigma-Aldrich.

\section{Preparation of Manihot esculenta aqueous leaf extract}

The leaves of Manihot esculenta were washed with tap water and then rinsed with deionized water to get rid of dust and particulate impurities. Afterwards, they were sun-dried and pulverized into fine powder to confer a large surface for absorption. Then, $48 \mathrm{~g}$ of the powder was mixed with $250 \mathrm{~mL}$ of deionized water in a $500 \mathrm{~mL}$ capacity conical flask. The mixture was sealed and boiled for $15 \mathrm{~min}$ in a thermostatic water bath. After allowing to cool to room temperature, it was filtered to obtain the aqueous extract. The extract was stored in a refrigerator between 5 and $10{ }^{\circ} \mathrm{C}$ for further use.

\section{Synthesis of MgONPs}

$250 \mathrm{~mL}$ of the Manihot esculenta aqueous leaf extract was added in drops to $50 \mathrm{~mL}$ of $0.1 \mathrm{M}$ solution of $\mathrm{Mg}\left(\mathrm{NO}_{3}\right)_{2} \cdot 6 \mathrm{H}_{2} \mathrm{O}$ in a $500 \mathrm{~mL}$ capacity beaker under a constant stirring condition with a magnetic stirrer. The total time consumed for the addition was $12 \mathrm{~h}$. The obtained solid-liquid dispersion was then centrifuged at $7000 \mathrm{rpm}$ for $10 \mathrm{~min}$. After this, the supernatant was discarded and the residue washed with deionized water to remove excess $\mathrm{Mg}\left(\mathrm{NO}_{3}\right) \cdot 6 \mathrm{H}_{2} \mathrm{O}$ and residual organic molecules. Furthermore, it was dried in an oven at $70{ }^{\circ} \mathrm{C}$ for $2 \mathrm{~h}$ and then calcined in a furnace at $500{ }^{\circ} \mathrm{C}$ for $3 \mathrm{~h}$ to obtain MgONPs. Finally, the obtained MgONPs were sonicated at $40{ }^{\circ} \mathrm{C}$ for $1 \mathrm{~h}$ in an ultrasonic cleaner (CLEAN-120HD) to disperse the particles.

\section{Characterization}

To identify the phytochemicals responsible for the reduction reaction and stabilization of the formed MgONPs, the Manihot esculenta aqueous leaf extract was analyzed in a gas chromatograph-mass spectrometer (GC-MS) (Shimadzu QP2010SE) at a 1:1 injection ratio of the ethanolic solution of the extract into the machine. Thereafter, the phytochemicals were identified based on their fragmentation patterns via a database incorporated into the GC-MS machine. MgONPs formation in the reaction mixture between $\mathrm{Mg}\left(\mathrm{NO}_{3}\right)_{2} \cdot 6 \mathrm{H}_{2} \mathrm{O}$ and the Manihot esculenta aqueous leaf extract was confirmed using UV-Vis absorption spectrophotometer (Uniscope SM 7504) in the wavelength between 100 and $700 \mathrm{~nm}$.

Morphological study to assess the microstructure, particle distribution and elemental composition of the MgONPs was performed in a scanning electron microscope (SEM) equipped with an energy-dispersive X-ray analyzer (EDX) 
unit (SEM: JEOL JSM 7660F). The sample was analyzed with an accelerating voltage of $15 \mathrm{kV}$.

The particle size and structure of the MgONPs were determined by transmission electron microscopy (TEM) (TEM: JEM-ARM200F-G) using $200 \mathrm{kV}$ accelerating voltage. Then, the average particle size was determined from the SEM and TEM micrographs using ImageJ software.

The diffraction pattern of the MgONPs was evaluated in an X-ray diffractometer (XRD) (XRD: Rigaku D/Max-IIIC) to study the type of phases present in the sample and to assess the crystalline structure of the MgONPs.

Fourier transform infrared (FTIR) spectroscopy (FTIR: Nicolet iS10) with wavenumber in the range $350-4000 \mathrm{~cm}^{-1}$ was employed to assess the type of bonds present in the MgONPs so as to complement and confirm the result of the analysis with GC-MS, UV and XRD.

\section{Results and discussion}

\section{UV-Vis spectrophotometry}

The optical properties of metal nanoparticles strongly depend on the size, shape and interaction between the particles present on the surface of the nanoparticles. The UV-Vis absorption spectrum of the MgONPs solution shows a sharp absorption band at $260 \mathrm{~nm}$ (Fig. 1) corresponding to the dipole resonance of $\mathrm{MgO}$ nanospheres, usually in the range 260-280 nm [7, 10]. The UV-Vis result clearly shows that the reductive biomolecules in the Manihot esculenta leaf extract were able to perform bio-reduction role leading to

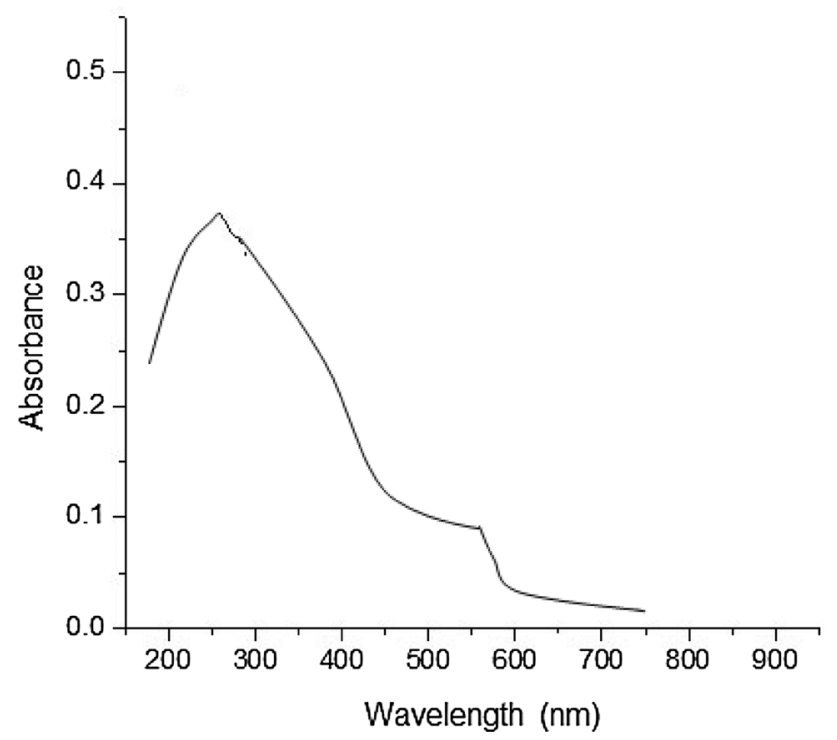

Fig. $1 \mathrm{UV}-\mathrm{Vis}$ spectrum of the colloidal solution of Manihot esculenta leaf extract and $\mathrm{Mg}\left(\mathrm{NO}_{3}\right)_{2} \cdot 6 \mathrm{H}_{2} \mathrm{O}$ showing maximum absorption at $260 \mathrm{~nm}$ due to the presence of MgONPs the formation of MgONPs. The biomolecules identified in high concentration from the GC-MS analysis include glycerin, D-mannitol, 2-deoxy-D-galactose, 11-(cyclopentene1-yl)undecanoic acid, [1,1'-bicyclopropyl]-2-octanoic acid, 9,9-dimethoxybicyclo [1,3] nona-2,4-dione, 2-methoxy4 -vynylphenol, $\beta$-sitosterol and cholest-5-en-3-ol. Characteristically, these molecules contain the functional groups $-\mathrm{C}-\mathrm{O}-\mathrm{C},-\mathrm{C}-\mathrm{O}-,-\mathrm{C}=\mathrm{C}-$ and $-\mathrm{C}=\mathrm{O}-$ which have been proposed to act as reducing agents in green synthesis [11].

\section{Microstructural characteristics and elemental composition of the MgONPs}

The morphology of the MgONPs is presented in Fig. 2. The microstructure shows hexagonal particles of average size $37.3 \mathrm{~nm}$ appearing as different ensembles. The particles showed very little degree of agglomeration and were well distributed over the surface of the material to present a large surface area-to-volume ratio. A previous study [12] obtained a bactericidal efficiency of $96.12 \%$ when the MgONPs size was $35.9 \mathrm{~nm}$, whereas at $47.3 \mathrm{~nm}$, the bactericidal efficiency was $94.46 \%$ against Bacillus subtilis var. niger, a kind of spore with dense coating and high resistance against chemicals. The same study also reported that when the particles were micron-sized $(2145.9 \mathrm{~nm})$, a much lower bactericidal efficiency of $75.71 \%$ was obtained.

The particles size of the MgONPs obtained in this study falls between $35.9-47.3 \mathrm{~nm}$, but closer to $35.9 \mathrm{~nm}$, hence should exhibit a comparable effect against Bacillus subtilis var. niger. Besides, the surface morphology exhibited by the MgONPs could be beneficial for catalysis application [13] as well as offer optical and other medicinal values

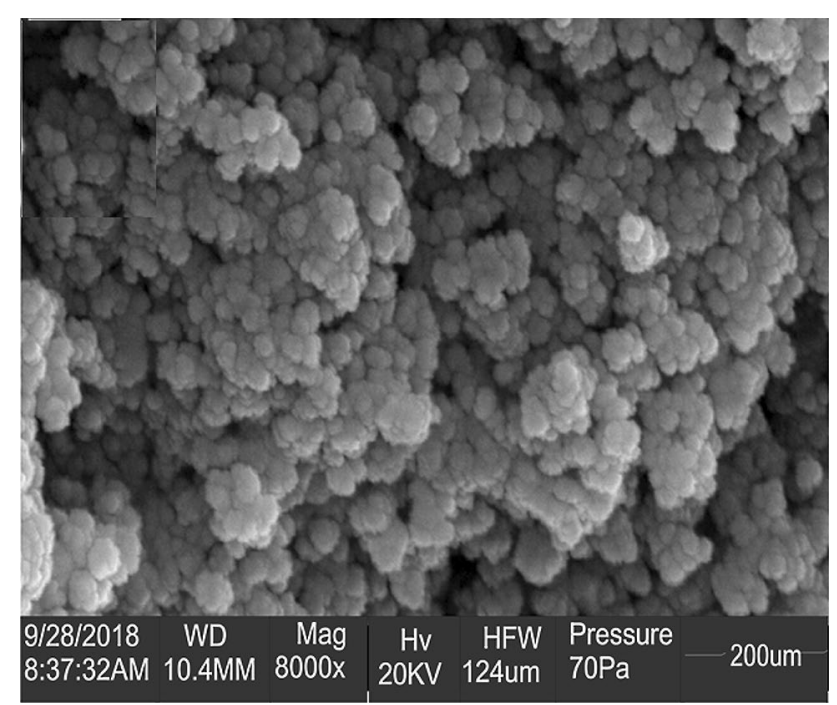

Fig. 2 SEM micrograph of the MgONPs showing well distributed particles 
[10]. The large surface area implies that in heterogeneous catalysis, the MgONPs should function as the metal [14], therefore, resulting in improved performance compared with the bulk material.

The formation of MgONPs was further confirmed by the EDX spectrum (Fig. 3), which shows the presence of $\mathrm{Mg}$ and $\mathrm{O}$ in the sample at high intensity and at 1:1 atomic ratio as found in pure $\mathrm{MgO}$ compound. The carbon atom observed in the spectrum is from the various organic biomolecules present in the plant extract which acted as reducing and capping agents during the reaction to form the MgONPs.

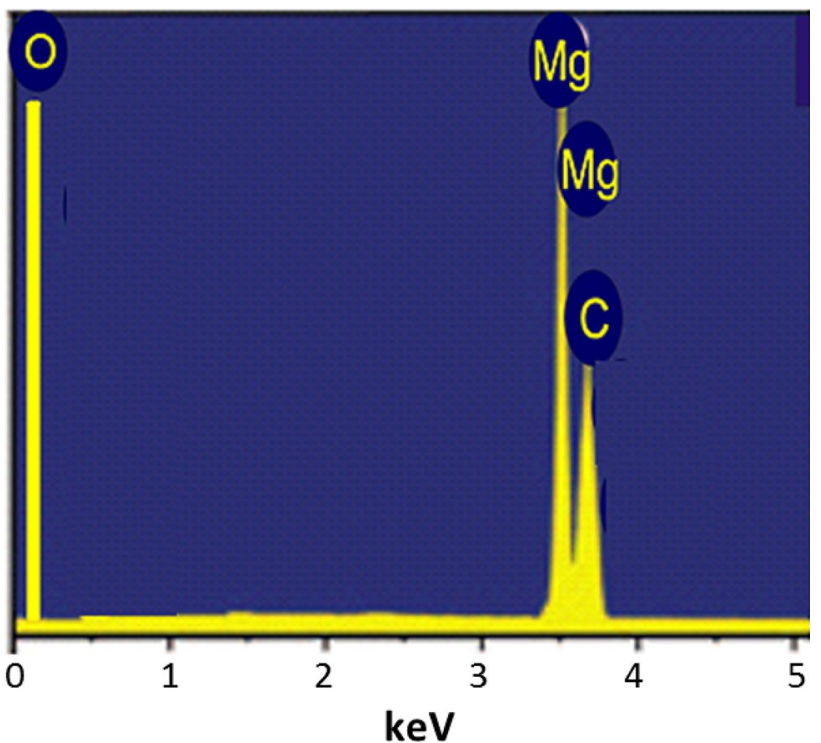

Fig. 3 EDX spectrum of the MgONPs showing the presence of $\mathrm{Mg}$ and $\mathrm{O}$ at $1: 1$ atomic ratio

\section{Particle shape and size}

The TEM assessment indicates that the particles were hexagonal in shape (Fig. 4). In addition, they were monodispersed with high size uniformity in the average of $36.7 \mathrm{~nm}$ (Fig. 4a). These properties were also observed in another TEM image of the sample viewed at a different magnification, presented in Fig. 4b. The hexagonal nature of the particles which was first observed in the SEM micrograph (Fig. 2), is more evident in the TEM micrographs, suggesting crystalline nature. Besides, the TEM result further supports the SEM morphology of the particles (Fig. 2), which showed particles with near-size uniformity. The ImageJassisted measurement of the average particle size of the MgONPs from the SEM micrograph (37.30 nm) compares with that obtained from the TEM (36.9 nm) micrograph, thus giving validating the size dimension of the MgONPs.

The properties exhibited by the MgONPs may be the result of the efficient capping ability of the phytochemicals present in the plant extract together with the ultrasonic radiation passed through the sample in ethanol medium, which improved dispersion and homogeneity of the particles [15]. Efficient dispersion of particles affects their interaction with test media in various in vitro and in vivo experiments, such as cytotoxicity [15] and in supported heterogeneous catalysis [16-18].

\section{Diffraction pattern of the MgONPs}

XRD evaluation of the synthesized MgONPs was conducted to examine the crystalline structure and phase purity of the sample. As observed in the diffractogram shown in Fig. 5, the diffraction pattern shows various peaks corresponding to (111), (200), (220), (311) and (222) reflection planes. The peaks match those in the standard reference file (JCDPS No.
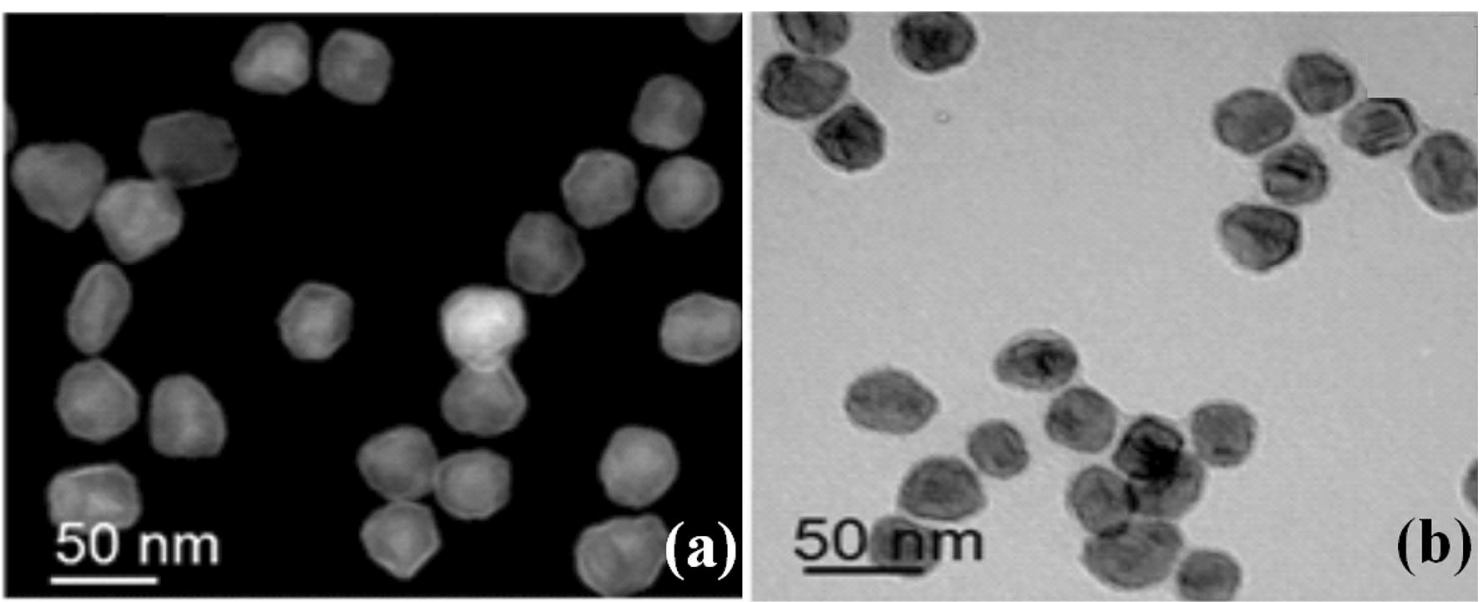

Fig. 4 TEM images of the MgONPs at different magnifications showing monodisperse particles 


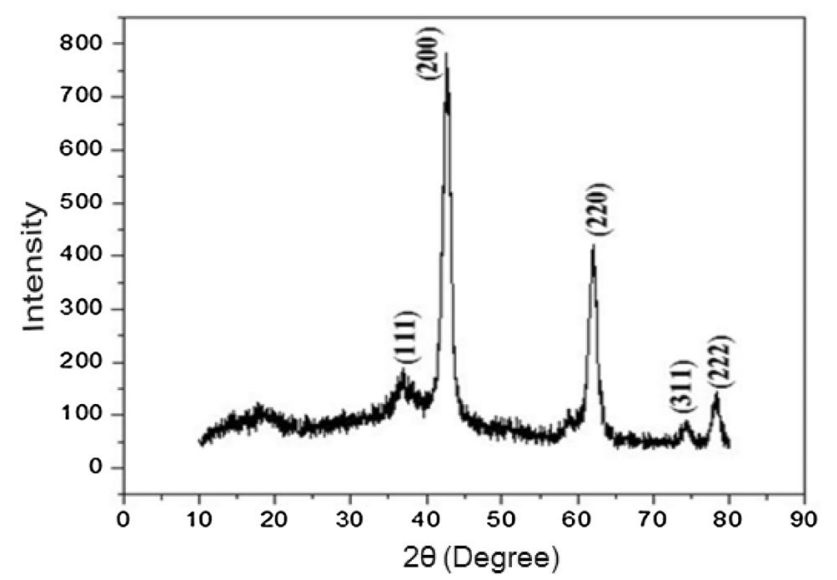

Fig. 5 XRD pattern of the MgONPs showing monophasic $\mathrm{MgO}$

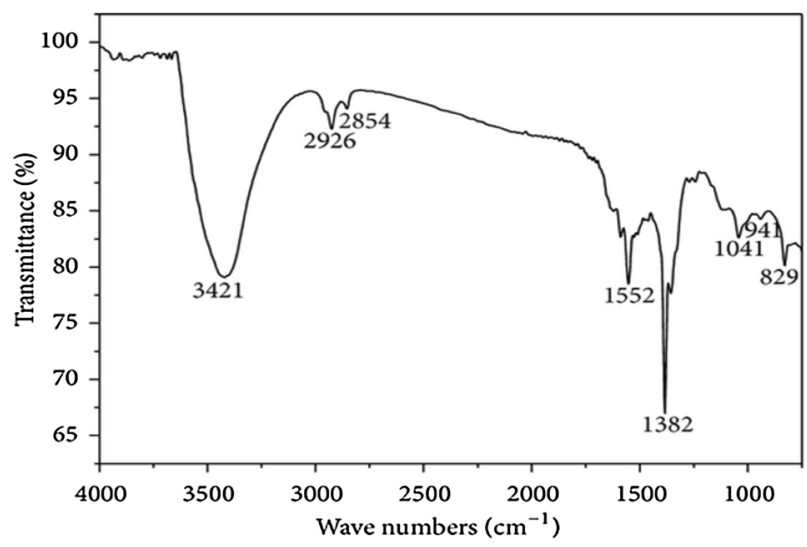

Fig. 6 FTIR spectrum of the synthesized MgONPs showing bonds related to the capping biomolecule contained in the $M$. esculenta leaf extract and $\mathrm{MgO}$

75-0447) in both angular location and intensity, confirming the formation of hexagonal phase of $\mathrm{MgO}$ [19], as indicated earlier in the SEM and TEM results (Figs. 2 and 4). Furthermore, no other peaks are observed in the spectrum, which indicates the absence of metallic impurities, and thus supports the EDX result (Fig. 3).

\section{Bond properties of the MgONPs}

The FTIR spectrum of the MgONPs depicted in Fig. 6 shows broad absorption band centred at $3421 \mathrm{~cm}^{-1}$ due to the stretching vibration of $\mathrm{O}-\mathrm{H}$ group in an alcohol [20], which results from the capping reaction of the hydroxyl group of the phytochemicals in the leaf extract with the $\mathrm{MgO}$ particle. The small double peaks located at 2926 and $2854 \mathrm{~cm}^{-1}$ are considered for $\mathrm{C}-\mathrm{H}$ stretching vibrations in $\mathrm{CH}_{2}$ group present in the phytochemicals. The sharp peak at $1552 \mathrm{~cm}^{-1}$ signifies the presence of $\mathrm{N}-\mathrm{H}$ bond resulting from bending vibrational mode in an aromatic amine. It is possible that the aromatic amine formed in the mixture due to reaction between alkynes and alkanes present in the $M$. esculenta leaf extract with the nitrate from the $\mathrm{Mg}\left(\mathrm{NO}_{3}\right)_{2} \cdot 6 \mathrm{H}_{2} \mathrm{O}$ precursor. This transformation is possible due to changes such as oxidation, reduction or degradation occurring in the mixture during the stage of MgONPs formation.

The prominent peak at $1382 \mathrm{~cm}^{-1}$ is assigned to $\mathrm{Mg}-\mathrm{O}$ vibration [21] while the small peaks located at 1041 and 941 are associated with $\mathrm{C}-\mathrm{O}$ and $\mathrm{C}-\mathrm{O}-\mathrm{C}$ diagnostic bonds, respectively [22]. The peak observed around $829 \mathrm{~cm}^{-1}$ indicates the formation of hexagonal MgONPs [21, 23, 24], in agreement with the SEM, TEM and XRD results.

\section{Conclusion}

Monodisperse $\mathrm{MgO}$ nanoparticles have been successfully synthesized based on the green approach using Manihot esculenta aqueous leaf extract. GC-MS identified several organic biomolecules proposed to act as reductant and capping agents to form the $\mathrm{MgO}$ nanoparticles. Analysis conducted using UV-Vis spectrophotometry, SEM/EDX, TEM, XRD and FTIR confirmed the formation of hexagonal shaped MgONPs of average diameter of $36.7 \mathrm{~nm}$. One of the significances of this study is the use of the Manihot esculenta plant-mediated route which makes the process economical and more ecologically friendly, compared to chemical synthesis. In addition, Manihot esculenta, because it is grown in many developing countries in large quantities, where the tubers serve as a source of cassava, a staple food, implies that the leaves would be widely available for a potential commercial scale preparation of MgONPs, since they do not serve as dependable source of vegetable. Finally, based on the protocol established in this study, it is possible to obtain monodisperse $\mathrm{MgO}$ nanoparticles with shape and size uniformity.

Acknowledgements The authors are grateful to the Department of Food and Chemical Sciences, Bells University of Technology, Ota for providing technical support for this work.

\section{Compliance with ethical standards}

Conflict of interest The authors report no conflict of interest.

Open Access This article is distributed under the terms of the Creative Commons Attribution 4.0 International License (http://creativeco mmons.org/licenses/by/4.0/), which permits unrestricted use, distribution, and reproduction in any medium, provided you give appropriate credit to the original author(s) and the source, provide a link to the Creative Commons license, and indicate if changes were made. 


\section{References}

1. Laurent, S., Forge, D., Port, M., Roch, A., Robic, C., Vander, E.L., Muller, R.N.: Magnetic iron oxide nanoparticles: synthesis, stabilization, vectorization, physicochemical characterizations, and biological applications. Chem. Rev. 110, 2574 (2010)

2. Wojcieszak, R., Gene, M.J., Eloy, P., Gaigneaux, E.M., Ruiz, P.: Supported Pd nanoparticles prepared by a modified water-in-oil microemulsion method. Stud. Surf. Sci. Catal. 175, 789-792 (2010)

3. Rao, K.G., Ashok, C.H., Venkateshwara, R.K., Chakra, C.H.S.: Structural properties of MgO NPs, synthesized by Co-precipitation technique. Int. J. Sci. Res. 3(12), 43-46 (2014)

4. Mohanraj, R.: Antimicrobial activities of metallic and metal oxide nanoparticles from plant extracts. In: Grumezescu, A.M. (ed.) Antimicrobial nanoarchitectonics from synthesis to applications, pp. 83-100. Elsevier, New York (2017)

5. Jhansi, K., Jayarambabu, N., Reddy, K.P., Reddy, N.M., Suvarna, R.P., Rao, K.V., Kumar, V.R., Rajendar, V.: Biosynthesis of MgO nanoparticles using mushroom extract:effect on peanut (Arachis hypogaea L.) seed germination. 3 Biotech. 7, 263 (2017)

6. Abdallah, Y., Ogunyemi, S.O., Abdelazez, A., Zhang, M., Hong, X., Ibrahim-Hossain, A., Fouad, H., Li, B., Chen, J.: The green synthesis of $\mathrm{MgO}$ nano-Flowers using Rosmarinus officinalis $\mathrm{L}$. (Rosemary) and the antibacterial activities against Xanthomonas oryzae pv. oryzae. BioMed Res. Int. 209, 5620989 (2019)

7. Vergheese, M., Kiran-Vishal, S.: Green synthesis of magnesium oxide nanoparticles using Trigonella foenum-graecum leaf extract and its antibacterial activity. J. Pharmacogn. Phytochem. 7, 1193$1200(2018)$

8. Thambi, M., Cherian, T.: Pesticidal activity of the leaves of Manihot esculenta against the pest Sitophilus oryzae. Pharma Innov. J. 4, 15-18 (2015)

9. Rahalison, L., Gupta, M.P., Santana, A.I.: Screening for antifungal activity of Panamanian plants. J. Pharmacol. 31, 68-76 (1993)

10. Moorthy, S.K., Ashok, C.H., Rao, K.V., Viswanathana, C.: Synthesis and characterization of $\mathrm{MgO}$ nanoparticles by neem leaves through green method. Mater. Today Proc. 2, 4360-4368 (2015)

11. Huang, J., Chen, C., He, N., Hong, J., Lu, Y., Qingbiao, L., Shao, W., Sun, D., Wang, X.H., Wang, Y., Yiang, X.: Biosynthesis of silver and gold nanoparticles by novel sundried Cinnamomum camphora leaf. Nanotechnology 18, 105-106 (2007)

12. Lei, H., Dianqing, L., Yanjun, L., Evans, D.G., Xue, D.: Influence of nano- $\mathrm{MgO}$ particle size on bactericidal action against Bacillus subtilis var, niger. Chin Sci. Bull. 50, 514-519 (2005)
13. Navalón, S., García, H.: Nanoparticles for catalysis. Nanomaterials 6, 123 (2016)

14. Astruc, D.: Nanoparticles and catalysis. Wiley, New York (2008)

15. Kaur, I., Ellis, L.-J., Romer, I., Tantra, R., Carriere, M., Allard, S., Mayne-L'Hermite, M., Minelli, C., Unger, W., Potthoff, A., Rades, S., Valsami-Jones, E.: Dispersion of nanomaterials in aqueous media: towards protocol optimization. J. Vis. Exp. 130, 56074 (2017)

16. Bell, A.T.: The impact of nanoscience on heterogeneous catalysis. Science 299, 1688-1691 (2003)

17. Boudart, M.: Catalysis by supported metals. In: Frankenburg, W.G. (ed.) Advances in catalysis, pp. 153-166. Academic Press, New York (1969)

18. Gates, B.C.: Supported metal clusters: synthesis, structure, and catalysis. Chem. Rev. 95, 511-522 (1995)

19. Safaei-Ghomia, J., Zahedia, S., Javida, M., Ghasemzadeh, M.A.: $\mathrm{MgO}$ nanoparticles: an efficient, green and reusable catalyst for the onepot syntheses of 2,6-dicyanoanilines and 1,3-diarylpropyl malononitriles under different conditions. J. Nanostruct. 5, 153-160 (2015)

20. Dobrucka, R.: Synthesis of $\mathrm{MgO}$ nanoparticles using Artemisia abrotanum herbal extract and heir antioxidant and photocatalytic properties. Iran J. Sci. Technol. Trans Sci. (2016). https://doi. org/10.1007/s40995-016-0076-x

21. Viswanatha, R., Venkatesh, T.G., Vidyasagar, C.C., Nayaka, A.: Preparation and Characterization of $\mathrm{ZnO}$ and $\mathrm{Mg}-\mathrm{ZnO}$ nanoparticle. Arch. Appl. Sci. Res. 4, 480-486 (2012)

22. Somanathan, T., Krishna, T., Saravanan, V.M., Kumar, V., Kumar, R.: $\mathrm{MgO}$ nanoparticles for effective uptake and release of doxorubicin drug: $\mathrm{pH}$ sensitive controlled drug release. J. Nanosci. Nanotechnol. 16, 9421-9431 (2016)

23. Burton, B.B., Goldstein, D.N., George, S.M.: Atomic layer deposition of $\mathrm{MgO}$ using Bis(ethylcyclopentadienyl)magnesium and $\mathrm{H}_{2}$ O. J. Phys. Chem. C 113, 1939-1946 (2009)

24. Pei, L.Z., Yin, W.Y., Wang, J.F., Chen, J., Fan, C.G., Zhang, Q.F.: Low temperature synthesis of magnesium oxide and spinel powders by a sol-gel process. Mater. Res. 13, 339-343 (2010)

Publisher's Note Springer Nature remains neutral with regard to jurisdictional claims in published maps and institutional affiliations. 